\title{
Temperature dependence of pulse-induced mechanoluminescence excitation in coloured alkali halide crystals
}

\author{
NAMITA RAJPUT*, S TIWARI ${ }^{\dagger}$ and B P CHANDRA ${ }^{\dagger}$ \\ Department of Post Graduate Studies and Research in Physics and Electronics, Rani Durgawati University, \\ Jabalpur 482 001, India \\ ${ }^{\dagger}$ Pt. Ravi Shanker Shukla University, Raipur 492 010, India
}

MS received 4 June 2004; revised 17 August 2004

\begin{abstract}
In practice, the relative efficiencies of different crystals are often determined under identical conditions of temperature and excitation. If the temperature of a crystal is increased or decreased with respect to room temperature, luminescence efficiency may get increased or decreased according to the composition of the crystal. When coloured crystals of $\mathrm{NaCl}, \mathrm{NaBr}, \mathrm{KCl}$ and $\mathrm{KBr}$ are excited by pulse-induced excitation at different temperatures, the mechanoluminescence (ML) intensity increases with temperature. The ML intensity of first peak, $I_{\mathrm{m} 1}$, second peak, $I_{\mathrm{m} 2}$ and the total ML intensity, $I_{\mathrm{T}}$, initially increase with temperature and then tend to attain an optimum value for a particular temperature of crystals. The ratio, $I_{\mathrm{m} 2} / I_{\mathrm{ml}}$, is found to increase with increasing temperature of the crystals. The expression derived on the basis of rate equations, are able to explain the temperature dependence of ML intensity on several parameters.
\end{abstract}

Keywords. Mechanoluminescence; dislocations; pulse-induced excitation; alkali halides; radiative recombination.

\section{Introduction}

Mechanoluminescence (ML), the phenomenon of cold light emission induced during mechanical deformation of solids, links the mechanical, spectroscopic, electrical, structural and other properties of solids. A large number of organic and inorganic crystals and amorphous solids exhibit the ML phenomenon (Longchambon 1925; Walton 1977; Zink 1978; Chandra 1985). On the basis of the deformation in solids needed for producing ML, we can classify ML into three types, viz. fracto-induced ML, plasticoinduced ML and elastico-induced ML.

It has been found that in the substances showing luminescence at room temperature, the luminescence is quenched at some higher temperature. On the other hand, many substances which are not luminescent at room temperature, show luminescence at low temperature. Therefore, studies on the temperature dependence of luminescence is very interesting, sometimes yielding information to understand the nature of the crystals and to determine the effective trap depth (Leverenz 1950; Curie 1963; Chandra et al 1983). However, comparison of temperature dependence of luminescence efficiency and decay rates gives information about the location of dissipative transition and permits the calculation of activation energies and frequency factors for these transition in certain cases. The present paper reports the effect of temperature on the

*Author for correspondence
ML produced by pulse-induced excitation of coloured alkali halide crystals of $\mathrm{NaCl}, \mathrm{NaBr}, \mathrm{KCI}, \mathrm{KBr}$ and $\mathrm{KI}$.

\section{Experimental set-up for ML measurement}

The technique similar to Bridgman method was used in the present investigation for the growth of pure alkali halide crystals. In this method, solid-liquid interface was achieved by variation of the temperature gradient by varying heater current. In this process the material was melted in a ceramic crucible and then cooled slowly through the melting point. Chemical of AR (analytical reagent) grade supplied by E. Merck company were used as starting materials. The firing was done using a tabular furnace supplied by M/s INDFUR, which controls the temperature within $\pm 10^{\circ} \mathrm{C}$. The temperature of the furnace was measured by a chromel-alumel thermocouple. For growing single crystal, the material (in powder form) was melted in crucible and cooled very slowly through the melting point down to room temperature. Grown crystals were taken out of the crucible and cleaved to proper size.

Circuit diagram of the arrangement used for measuring ML activity is shown in figure 1 . The ML was excited by pressure pulse generated by a pulse generator designed for this purpose. A basic monostable multivibrator circuit using IC555 is designed for the generation of pressure pulses of $50 \mu$ s duration. The switching time can be varied by adjusting the value of $50 \mathrm{~K}$ resistor connected at pin nos 6 and 7 of IC555. The output of 555 is connected to 


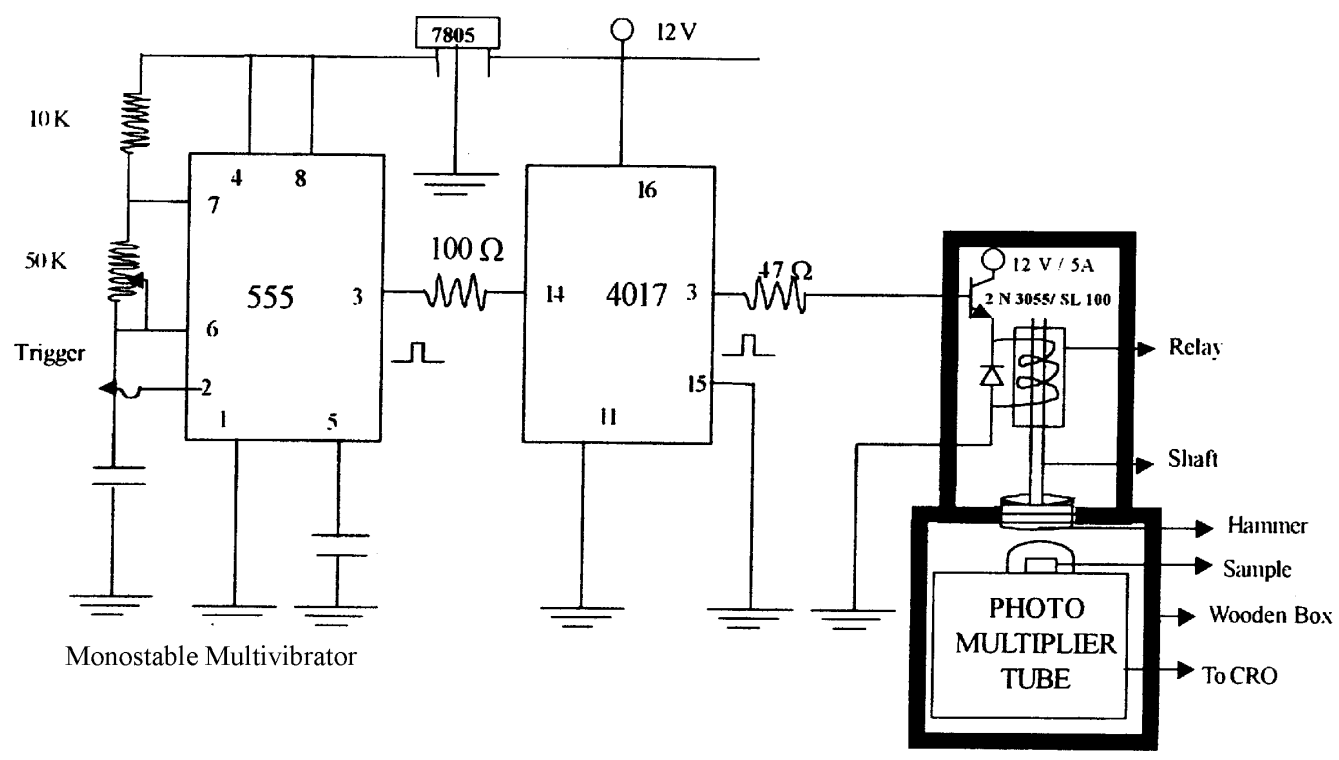

Figure 1. Circuit diagram of pulse-induced mechanoluminescence measuring instrument.

decade counter CD4017 and its output is connected to transistor SL100 which controls the relay and a high power electromagnet specially made for the proposed system is connected to the relay so that it impacts different strength induced ML in crystal. The output of the device is connected to hammer. When a signal trigger is applied to the input of the circuit device it produces electric pulse. This electric energy is converted into mechanical energy by the hammer connected at the output of the device.

For measuring the effect of temperature on ML, the crystals were placed onto a Lucite plate and heated by using two heating filaments (35 watt each) fixed near to it. By changing the voltage applied to the filament, the crystals could be heated at different temperatures. In the present study, the temperature range was from room temperature to $120^{\circ} \mathrm{C}$. The $\mathrm{ML}$ was excited during the impact of a moving hammer onto the crystal (Chandra et al 1980a,b,c) and the luminescence was recorded by a RCA931A photomultiplier tube (PMT) placed just below the lucite plate. The output of the PMT was fed to the dual beam oscilloscope having P7 phosphorescent screen capable of sustaining a trace in dark for more than a minute. The size of the crystals used for present investigation were $1 \times 1 \times$ $1 \mathrm{~mm}^{3}, 2 \times 2 \times 2 \mathrm{~mm}^{3}, 3 \times 3 \times 3 \mathrm{~mm}^{3}, 4 \times 4 \times 4 \mathrm{~mm}^{3}$. At least four crystals were studied for each set of observations. The temperature of the crystal was measured by a calibrated chromel-alumel thermocouple.

\section{Results}

During pulse-induced excitation of $\gamma$-irradiated alkali halide crystals, two peaks are observed in the ML intensity versus time curves. The first peak lies in the deformation region and the second peak in the post deformation re-

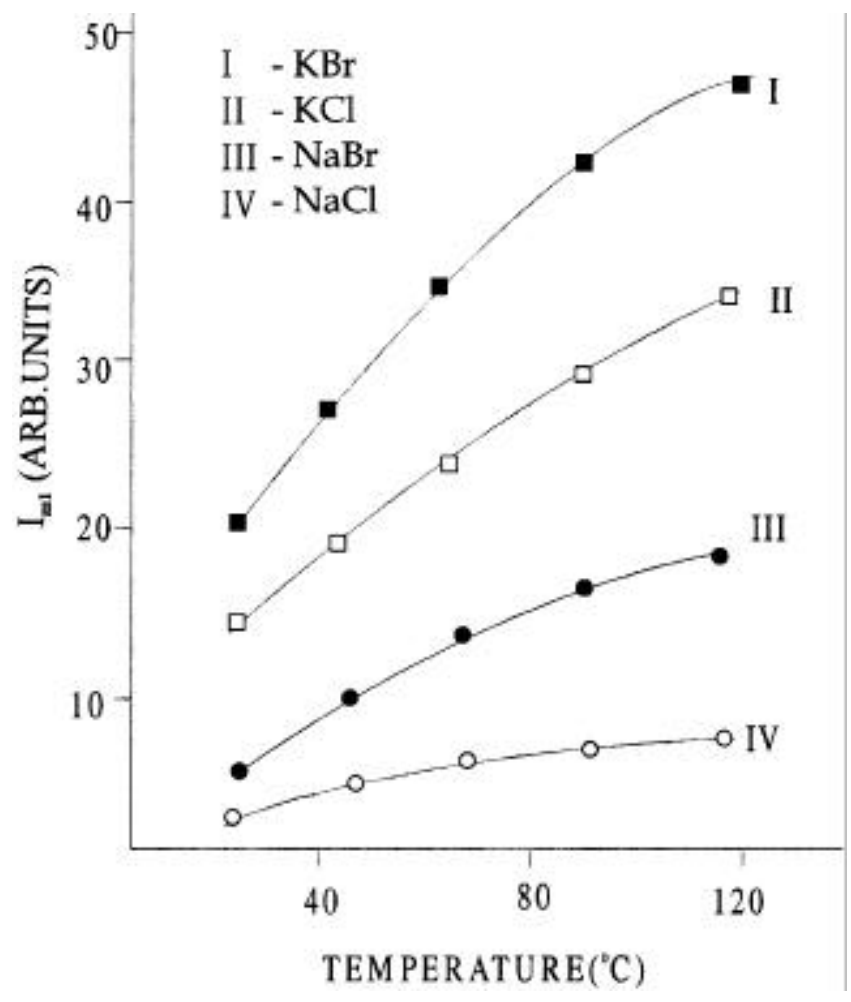

Figure 2. Dependence of the peak ML intensity, $I_{\mathrm{m} 1}$, on temperature of $\gamma$-irradiated alkali halide crystals.

gion. The ML in the deformation region is due to the recombination of dislocation trapped electrons with the holes in defect centres. The ML in the post deformation region is due to the transient thermostimulated luminescence from shallow traps which get populated due to the Auger process occurring during transfer of dislocation trapped electrons to deep traps. $I_{\mathrm{m} 1}, I_{\mathrm{m} 2}$ are the intensities 
corresponding to first and second peak, respectively in the ML intensity versus time curve and $I_{\mathrm{T}}$ is the total ML intensity, i.e. the integrated area below this curve.

Figures 2-4 show the dependence of $I_{\mathrm{m} 1}, I_{\mathrm{m} 2}$ and $I_{\mathrm{T}}$, respectively, on temperature of coloured $\mathrm{NaCl}, \mathrm{NaBr}, \mathrm{KCl}$ and $\mathrm{KBr}$ crystals. It is found that $I_{\mathrm{m} 1}, I_{\mathrm{m} 2}$ and $I_{\mathrm{T}}$ initially increase with temperature and then attain an optimum value for a particular temperature of the crystals.

Figure 5 shows the plot of $I_{\mathrm{m} 2} / I_{\mathrm{m} 1}$ with temperature for coloured alkali halide crystals. It is found that the ratio, $I_{\mathrm{m} 2} / I_{\mathrm{m} 1}$, increases with increasing temperature of the crystals.

Figures 6 and 7 show the dependence of $I_{\mathrm{m} 1}, I_{\mathrm{m} 2}$ and $I_{\mathrm{T}}$ on temperature for coloured KI crystals. It is found that $I_{\mathrm{m} 1}, I_{\mathrm{m} 2}$ and $I_{\mathrm{T}}$ decrease with increasing temperature of $\mathrm{KI}$ crystals.

\section{Discussion}

Consider a crystal having length, $L$, breadth, $W$ and thickness, $H$. If the crystal is deformed along the plane parallel to its breadth side, then the rate of creation of new surfaces is given by $2 W v$, where $v$ is the average velocity of crack propagation.

It is known that a large number of moving dislocations are generated near the tip of moving cracks. If $B$ is the cor-

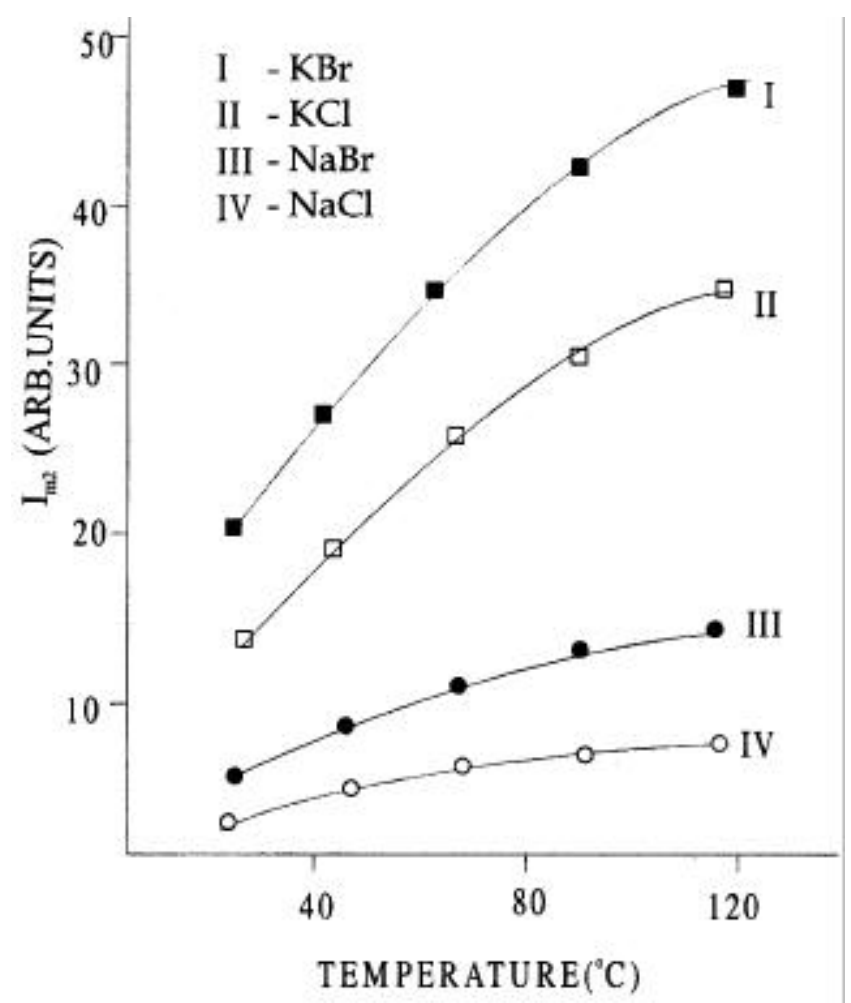

Figure 3. Dependence of the peak ML intensity, $I_{\mathrm{m} 2}$, on temperature of $\gamma$-irradiated alkali halide crystals. relation factor between the rate of generation of moving dislocations and rate of generation of new surfaces, then the rate of generation of moving dislocations may be written as

$$
G_{\mathrm{d}}=2 B W v .
$$

If $r_{\mathrm{F}}$ is radius of interaction between the moving dislocations and $F$-centre, $\lambda$ the mean free path of moving dislocations, $n_{\mathrm{F}}$ the density of F-centres and $p_{\mathrm{F}}$ the probability of capture of F-centre electrons by the moving dislocations, then the rate of generation of dislocation electrons may be expressed as (Chandra 1996)

$$
g=2 \lambda p_{\mathrm{F}} r_{\mathrm{F}} n_{\mathrm{F}} B W v / \beta^{\prime}\left[1-\exp \left(-\beta^{\prime} t\right)\right],
$$

here $\beta^{\prime}=1 / \tau_{\mathrm{i}}$ and $\tau_{\mathrm{i}}$ the pinning time of moving dislocations.

On the basis of (2) we shall discuss the characteristics of ML in coloured alkali halide crystals.

\subsection{Kinetics of the transient $M L$}

For $\beta^{\prime} t<1$, (2) may be written as

$$
g=2 \lambda p_{\mathrm{F}} r_{\mathrm{F}} n_{\mathrm{F}} B W v t .
$$

The electrons captured by moving dislocations, move with them and they encounter with the defect centres like hole centres, deep traps, stationary dislocations and other

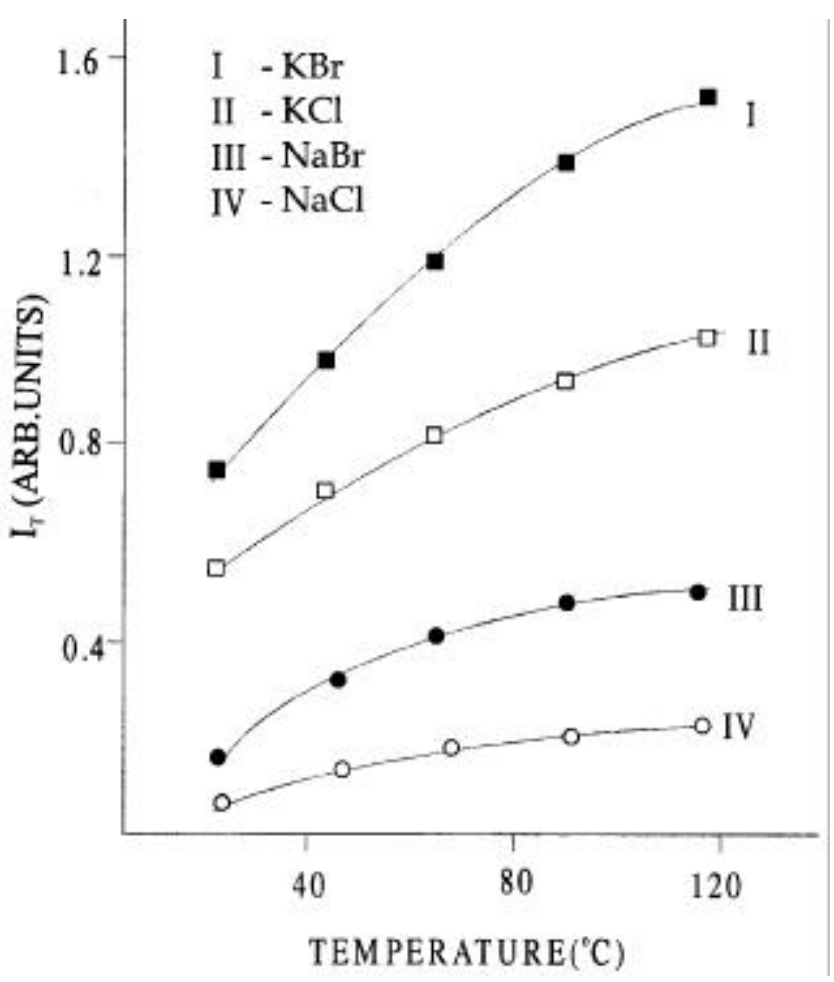

Figure 4. Dependence of the total ML intensity, $I_{\mathrm{T}}$, on temperature of $\gamma$-irradiated alkali halide crystals. 
compatible traps, they may be captured by these defect centres. If $\sigma_{1}, \sigma_{2}, \sigma_{3}$ and $\sigma_{4}$ are the cross-sections, $N_{1}, N_{2}$, $N_{3}$ and $N_{4}$ are the densities of the hole centres, deep traps, stationary dislocations states, and other compatible traps, respectively and $\eta$ is the probability of radiative recombination of moving dislocation electrons with hole centres, then the time dependence of the transient ML intensity may be expressed as

$$
\begin{aligned}
& I_{\mathrm{r}}=2 \eta \sigma_{1} N_{1} \lambda p_{\mathrm{F}} r_{\mathrm{F}} n_{\mathrm{F}} B W v t / \\
& \left(\sigma_{1} N_{1}+\sigma_{2} N_{2}+\sigma_{3} N_{3}+\sigma_{4} N_{4}\right) .
\end{aligned}
$$

The velocity of crack propagation may be given by

$$
v=H / t_{\mathrm{ml}} \text {. }
$$

\subsection{Estimation of $I_{m 1}$}

From (4) and (5), the value of first peak ML intensity, $I_{\mathrm{ml}}$ at $t=t_{\mathrm{m}}$ may be given by

$$
\begin{aligned}
& I_{\mathrm{ml}}=\eta \sigma_{1} N_{1} \lambda p_{\mathrm{F}} r_{\mathrm{F}} n_{\mathrm{F}} B A / \\
& \left(\sigma_{1} N_{1}+\sigma_{2} N_{2}+\sigma_{3} N_{3}+\sigma_{4} N_{4}\right),
\end{aligned}
$$

where $A=2 W H$ is the area of the newly created surfaces of the crystal.

\subsection{Estimation of $I_{m 2}$}

The delayed ML is actually due to the recombination of electrons moving in the stationary dislocation band with the hole centres. If $\gamma_{\mathrm{s}}=1 / \tau_{\mathrm{s}}$ and $\tau_{\mathrm{s}}$ is the life time of elec-

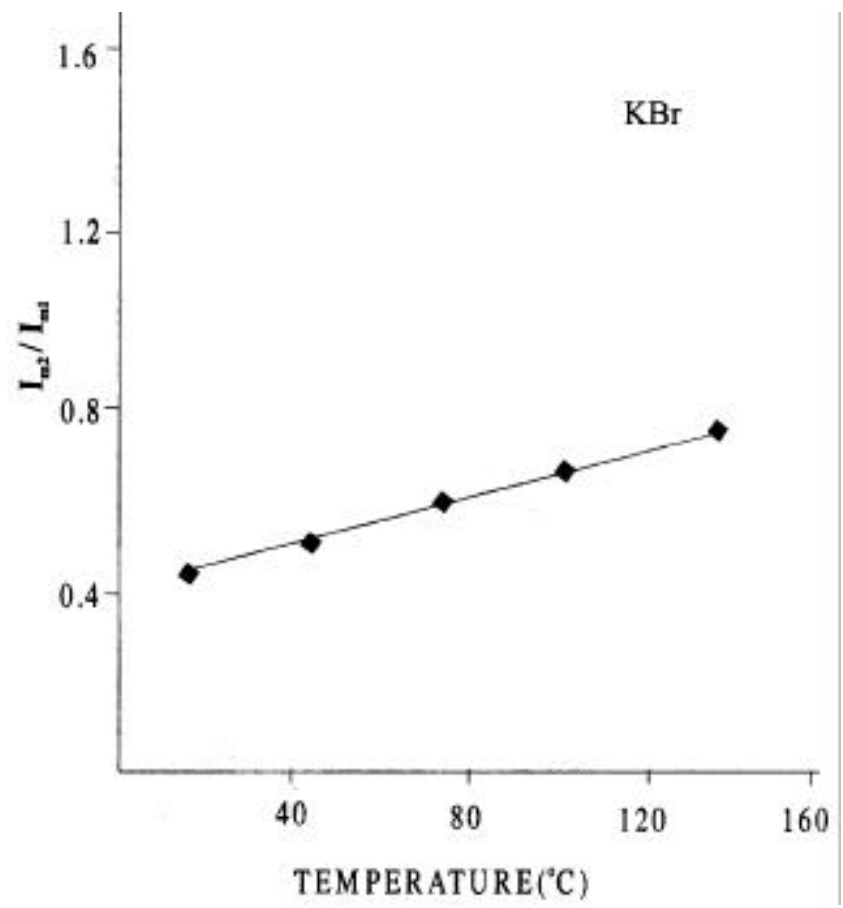

Figure 5. Dependence of $I_{\mathrm{m} 2} / I_{\mathrm{m} 1}$ on temperature for $\gamma$-irradiated alkali halide crystals. trons in the stationary dislocation band, the value of second peak ML intensity, $I_{\mathrm{m} 2}$, may be given by

$$
\begin{aligned}
& I_{\mathrm{m} 2}=\eta \sigma_{3} N_{3} \lambda p_{\mathrm{F}} r_{\mathrm{F}} n_{\mathrm{F}} B \gamma_{\mathrm{s}} A / \\
& \beta^{\prime}\left(\sigma_{1} N_{1}+\sigma_{2} N_{2}+\sigma_{3} N_{3}+\sigma_{4} N_{4}\right) .
\end{aligned}
$$

It is evident from (6) and (7) that both $I_{\mathrm{ml}}$ and $I_{\mathrm{m} 2}$ depend directly on $\eta, p_{\mathrm{F}}$ and $n_{\mathrm{F}}$. At low temperature, $\eta$ and $n_{\mathrm{F}}$ remain nearly constant and therefore, $I_{\mathrm{ml}}$ and $I_{\mathrm{m} 2}$ should increase with increasing temperature of the crystals. On the other hand, at higher temperature, $n_{\mathrm{F}}$, decreases significantly due to the thermal bleaching of colour centres. Thus, in the higher temperature range, $I_{\mathrm{ml}}$ and $I_{\mathrm{m} 2}$ should decrease with increasing temperature of the crystals. Thus, both $I_{\mathrm{ml}}$ and $I_{\mathrm{m} 2}$ should be optimum for a particular temperature of the crystals.

\subsection{Estimation of total ML intensity, $I_{\mathrm{T}}$}

The total ML intensity, $I_{\mathrm{T}}$, i.e. the integrated area below the ML intensity versus time curve, may be given by

$$
\begin{aligned}
& I_{\mathrm{T}}=\int_{0}^{\infty} I \mathrm{~d} t \\
& I_{\mathrm{T}}=\eta \lambda p_{\mathrm{F}} r_{\mathrm{F}} n_{\mathrm{F}} B\left(\sigma_{1} N_{1}+\sigma_{3} N_{3}\right) A / \\
& \beta^{\prime}\left(\sigma_{1} N_{1}+\sigma_{2} N_{2}+\sigma_{3} N_{3}+\sigma_{4} N_{4}\right) .
\end{aligned}
$$

The total ML intensity, $I_{\mathrm{T}}$, depends directly on $\eta, p_{\mathrm{F}}$ and $n_{\mathrm{F}}$ and it depends inversely on the rate constant for the pinning of dislocation i.e. $\beta^{\prime}$. Since $p_{\mathrm{F}}$ increases with increasing temperature of the crystals and $\eta, n_{\mathrm{F}}$ and $1 / \beta^{\prime}$

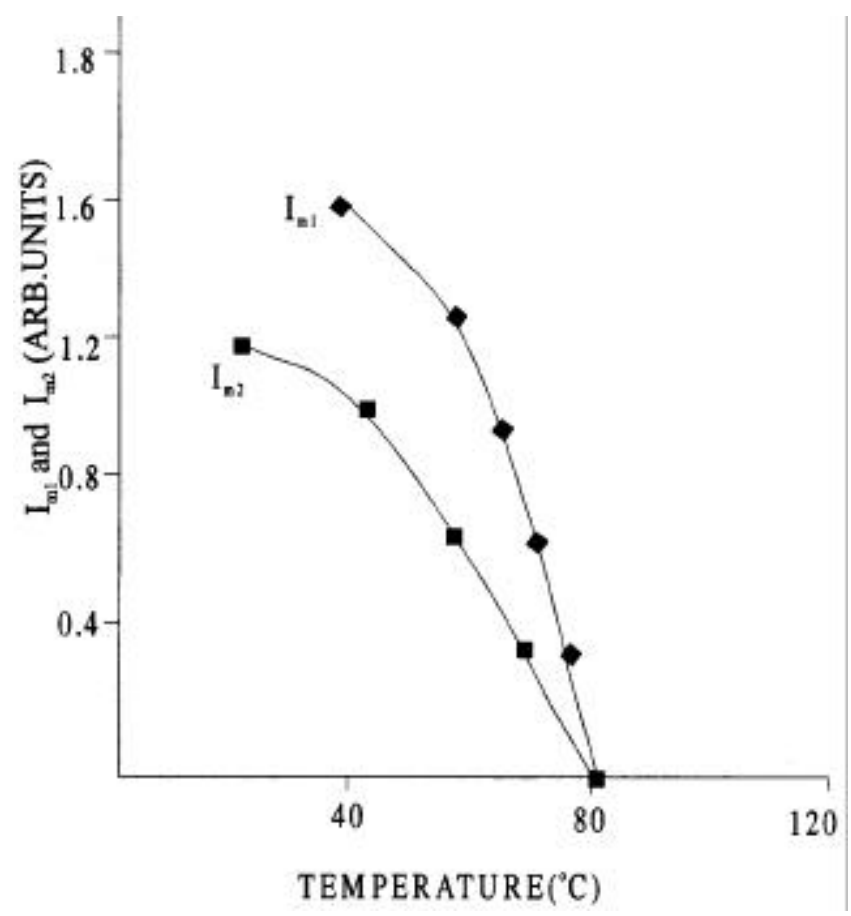

Figure 6. Dependence of the peak ML intensity, $I_{\mathrm{m} 1}$ and $I_{\mathrm{m} 2}$, on temperature of irradiated KI crystals. 


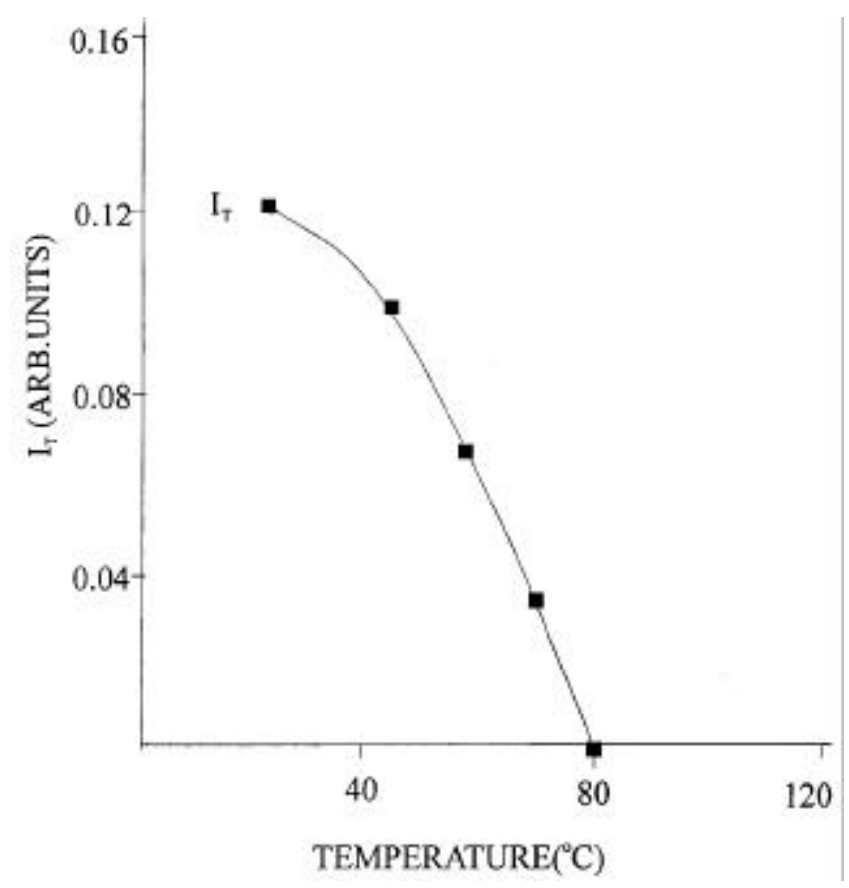

Figure 7. Dependence of the total ML intensity, $I_{\mathrm{T}}$, on temperature of irradiated KI crystals.

decrease with increasing temperature of the crystals, the total ML intensity, $I_{\mathrm{T}}$, should also be optimum for a particular temperature of the crystals. It is to be noted that whereas $I_{\mathrm{m} 1}$ and $I_{\mathrm{m} 2}$ depend only on $\eta, p_{\mathrm{F}}$ and $n_{\mathrm{F}}, I_{\mathrm{T}}$ depends on $\eta, p_{\mathrm{F}}, n_{\mathrm{F}}$ and $1 / \beta^{\prime}$. Thus in higher temperature range, $I_{\mathrm{T}}$ should decrease with a faster rate as compared to the decrease of $I_{\mathrm{m} 1}$ and $I_{\mathrm{m} 2}$ with increasing temperature of the crystals.

\subsection{Ratio of $I_{\mathrm{m} 1}$ and $I_{\mathrm{m} 2}$}

From (6) and (7), we get

$$
I_{\mathrm{m} 2} / I_{\mathrm{m} 1}=\sigma_{3} N_{3} \gamma_{\mathrm{s}} / \sigma_{1} N_{1} \beta^{\prime} .
$$

It is evident from (9) that the ratio of $I_{\mathrm{m} 2} / I_{\mathrm{m} 1}$ depends on the ratio of $N_{3}$ and $N_{1}$. Since there is no significant change in $N_{3}, N_{1}$ decreases with increasing temperature of the crystals, it seems that the ratio, $I_{\mathrm{m} 2} / I_{\mathrm{m} 1}$, should increase with increasing temperature of the crystals.

\section{Conclusions}

The main conclusions of the present investigation are as follows:

(I) The peak ML intensities, $I_{\mathrm{m} 1}, I_{\mathrm{m} 2}$ and the total $\mathrm{ML}$ intensity, $I_{\mathrm{T}}$, of coloured alkali halide crystals during pulseinduced deformation increase with increasing temperature, and attain an optimum value for a particular temperature of the crystal.

(II) The ratio, $I_{\mathrm{m} 2} / I_{\mathrm{m} 1}$, increases with increasing temperature of the coloured crystals of $\mathrm{NaCl}, \mathrm{NaBr}, \mathrm{KCl}$ and $\mathrm{KBr}$ during their pulse-induced deformation. This is due to the fact that $N_{1}$ i.e. the density of hole centres, decreases with increasing temperature of the crystals.

(III) It seems that for irradiated KI crystals, the temperature at which $I_{\mathrm{m} 1}, I_{\mathrm{m} 2}$ and $I_{\mathrm{T}}$ should attain an optimum value lies below the room temperature. Therefore, a decrease in $I_{\mathrm{m} 1}, I_{\mathrm{m} 2}$ and $I_{\mathrm{T}}$ is observed with increasing temperature of KI crystals. When the ML of irradiated KI crystals is measured at low temperature, the increase of $I_{\mathrm{m} 1}, I_{\mathrm{m} 2}$ and $I_{\mathrm{T}}$ is observed with increasing temperature of the crystals.

(IV) The effect of temperature on pulse-induced ML of coloured alkali halide crystals may be due to the fact that at lower temperature, the dislocation capture probability of F-centre electrons i.e. $p_{\mathrm{F}}$, increases with increasing temperature whereas at higher temperature the F-centre density, $n_{\mathrm{F}}$, decreases due to thermal bleaching.

\section{References}

Chandra B P 1985 Nuclear Tracks 10825

Chandra B P 1996 Radiat. Eff. \& Def. Solids 138119

Chandra B P and Zink J I 1980a Phys. Rev. B21 816

Chandra B P and Zink J I 1980b J. Chem. Phys. 735933

Chandra B P and Zink J I 1980c Inorg. Chem. 193098

Chandra B P, Elyas M and Majumdar B 1983 Solid State Commun. 42753

Curie D 1963 Luminescence in crystals (NY: John Wiley \& Sons) Leverenz H W 1950 An introduction to luminescence of solids (NY: John Wiley \& Sons Inc.)

Longchambon H 1925 Bull. Soc. Fr. Min. 40130

Walton A J 1977 Adv. Phys. 26887

Zink J I 1978 Acc. Chem. Res. 11289 